\title{
Prediction of the residual stress after quenching of 6061 aluminium alloy plates by using mathematical modelling
}

\author{
Marin Petre ${ }^{1, *}$, Cristian Dinu ${ }^{2}$, Nicuşor Constantin Drăghici ${ }^{1}$ and Valeriu Andrei ${ }^{1}$ \\ ${ }^{1}$ ALRO, 116 Piteşti Street, Slatina 230104, Romania \\ ${ }^{2}$ Department of Computer Science, University of Craiova, 13 A.I. Cuza, 200585, Romania
}

\begin{abstract}
The purpose of this article is to better understand the behavior of the residual stresses in aluminium alloy plates by using mathematical modelling. Quenching of aluminium alloy plates causes an uneven temperature variation in aluminum alloy plates, and elastic and elasto-plastic deformations occur inside the material. The latter causing the formation of deformations and residual stresses. The non-linear thermo-mechanical direct coupled analysis of the quenching process for a 6061 aluminium alloy plate was achieved by using ANSYS finite element software. The residual stresses due to solid thermal effects were determined by calculation of the Third principal stresses, the most negative or compressive. The developed mathematical model offers a support in the understanding the behavior of the residual stresses in aluminium alloy plates and a better control of them.
\end{abstract}

\section{Introduction}

The solution heat treatment and quenching operation of aluminium alloys induces internal stresses of tension and compression in the section of the plates due to heating in very high temperature ranges, near the lower limit of the melting intervals of aluminum alloys, and sudden cooling by water spraying on the upper and lower surfaces of the plates that determine thermal gradients in the range of $50-100 \mathrm{r} \mathrm{C} / \mathrm{s}$.

One of the most important steps in the mathematical modeling of an industrial process is to understand the process. In our case, in the initial quenching stage, there is a cooling of the plate from the outside to the inside, which causes a contraction and strengthening of the outer part of the plate, before the inner part, which causes internal compression stresses and the balance of the external stresses. After a short period of time, of the order of seconds, the inside of the plate begins to cool and contract. However, the contraction process is limited by the already quenched outer layer of the plate, which causes internal tensile stresses and which will be balanced by the external compression stresses [1]. The typical residual stresses after quenching process follow an M-shaped profile [2].

In this work, the quenching process by water spraying was modeled, which offers the advantage of time control as well as pressure regulation. The object of the mathematical model was a 6061 aluminium alloy plate. Aluminium alloy 6061 is a medium to high strength heat-treatable alloy. The magnesium and silicon are the major alloying elements of the 6061 aluminium alloy, which is one of the most common aluminum alloys for general-purpose us.

\footnotetext{
*e-mail: mapetre@alro.ro
} 
Quenching is a multi-physics process which involves heat transfer, phase transformation and stress evolution problems [3]. In the implemented mathematical model the structural stress due to solid thermal effects was considered, and the phase transformations that occur during quenching are not taken into account.

Numerous researches studies have been conducted in last decades to develop numerical simulation methods for the prediction of the residual stresses of the material after quenching [4].

The main challenge for this problem was to define the boundary conditions, respectively the heat transfer coefficients on the plate surface during quenching.

Considering the data of the problem and the mathematical model developed in earlier work [5] by using ANSYS Discovery AIM software, it was succeeded the prediction of the residual stress after quenching for a 6061 aluminium alloy plate.

\section{The mathematical model}

The mathematical model developed and implemented using the ANSYS software is presented in the a previous work [5].The object of the mathematical model was a 6061 aluminium alloy plate with the dimensions $100 \times 1500 \times 3000 \mathrm{~mm}$.

The properties of the 6061 aluminium alloy plate used as input data in the model were taken from the academic literature [6] and standards [7], and some of them interpolated by using the least square method [8].

By choosing the type of problem, ANSYS has already implemented the main equations of the mathematical model, in our case the transient heat transfer problem [5]. In fact, one of the most difficult parts in ANSYS is to define the boundary conditions of the studied problem.

The initial condition for the heat problem was:

$$
T_{\text {ini }}=550^{\circ} \mathrm{C}, \text { when } t=0 \mathrm{~s} .
$$

The boundary condition for the heat transfer problem was defined as follows:

$$
-\boldsymbol{n} \cdot(-k \nabla T)=q(A, t)
$$

Where: $\mathrm{k}$ is the thermal conductivity of the aluminium alloy plate, $\mathrm{T}$ temperature and $\mathrm{q}$ the heat flux coefficient dependent on the position of point $\mathrm{A}$ on the surface of the plate and the time $\mathrm{t}$.

For determination of the residual stresses on the studied aluminium alloy plate, the temperature value resulted from the heat problem was used as the input data in the structural problem.

Due to the assumption that the phase transition does not change during the quenching process, the thermal strain depends in our study only on the temperature, T, the stress-free initial temperature, $T_{\mathrm{ini}}$, and the thermal expansion vector, $a$ :

$$
\varepsilon_{t h}=\alpha\left(T-T_{\text {ini }}\right)
$$

Also, the zero state is considered as the initial state since the rolling operation before the quenching has minor effects on the residual stresses [9]. Thus, the strain consists only of elastic $\left(\varepsilon_{e l}\right)$ and thermal $\left(\varepsilon_{t h}\right)$ contributions:

$$
\varepsilon=\varepsilon_{e l}+\varepsilon_{t h}
$$

And the stress was related to the strain by: 


$$
\sigma=E \varepsilon
$$

The bottom of the plate was fixed in the four corners (points) on the transversal direction of the plate and free in the plane area.

\section{Experimental}

A part of the experiments were performed on the special equipment for the research of the aluminium alloy quenching process (Figure 1 - 2) and provided with the latest technology and commissioned in January 2019 into R\&D Department of the ALRO plant in Romania.

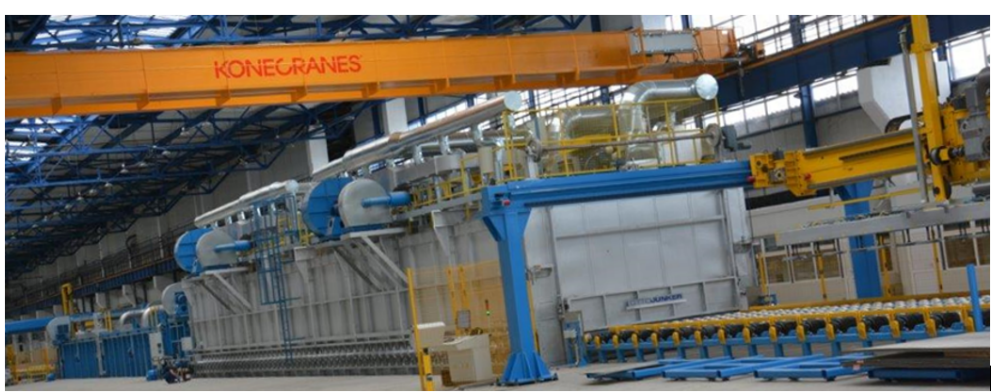

Figure 1. The independent equipment for the research of aluminium alloy quenching process.

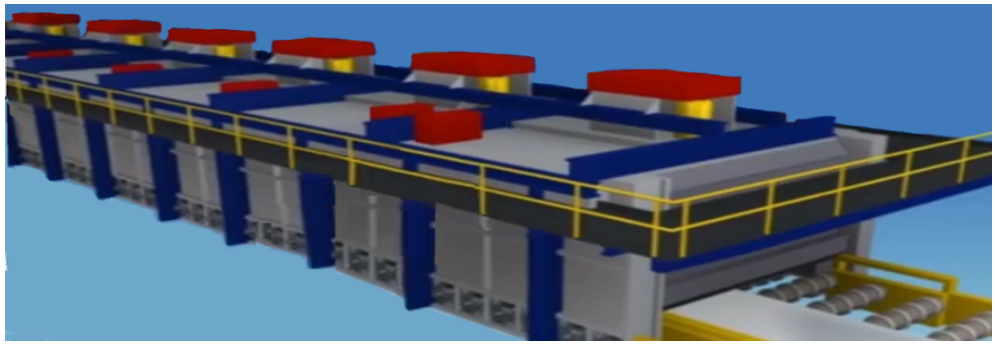

Figure 2. The independent equipment for the research of aluminium alloy quenching process 3D view.

The industrial tests were performed on 6061 aluminium alloy plates. The 6061 aluminium alloy plates were obtained by hot rolling from a cast slab.

At $t=0 \mathrm{~s}$, the plate was assumed to be entirely in the heat treatment area and the plate temperature was assumed to be $550 \mathrm{r} \mathrm{C}$, taking into consideration the temperature set points for this treatment. The plate was assumed to come into direct contact with the cooling medium after $1 \mathrm{~s}$. In our case the cooling medium was represented by demineralized water whose temperature is automatically controlled and is usually in the range of $25-35 \check{\text { r } ~}$.

The plates remain in the furnace for a certain period of time and at a certain temperature depending on their thickness and the type of alloy. After this period, the furnace door practically opens and the plates exit from the furnace by the movement induced by the conveying rollers directly in the quenching area.

After the quenching treatment, samples were cut at dimensions $300 \mathrm{~mm}$ x $300 \mathrm{~mm}$ for determination of the residual stresses by the hole-drilling method. By this method the residual 
stress profiles is determined near the surface, on $1 \mathrm{~mm}$ depth. Induction of the internal stresses during the drilling was avoided by utilization of a high-speed air turbine with $40000 \mathrm{rpm}$. Residual stresses were evaluated by Integral Method [10].

\section{Results. Discussion}

The mathematical was implemented using the commercial finite element package ANSYS Discovery AIM 19.0. Considering that inside the material the residual stresses are different from those on the outer surface, their representation was made on a quarter of the plate for a better visualization (Figure 3 ).

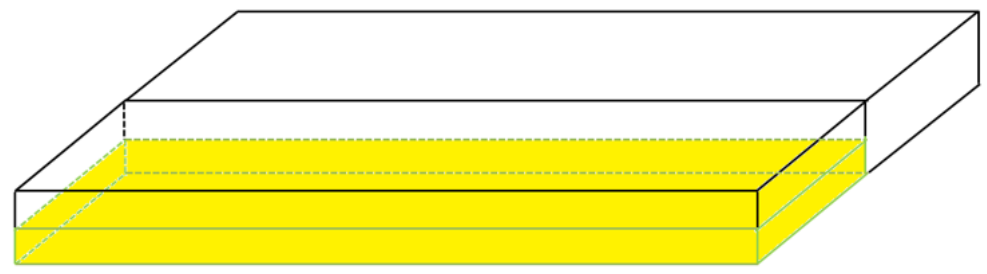

Figure 3. The computational domain for residual stresses represented with yellow color.

The residual stresses on the surfaces of a quarter of the plate after the quenching process are presented in Figure 4.

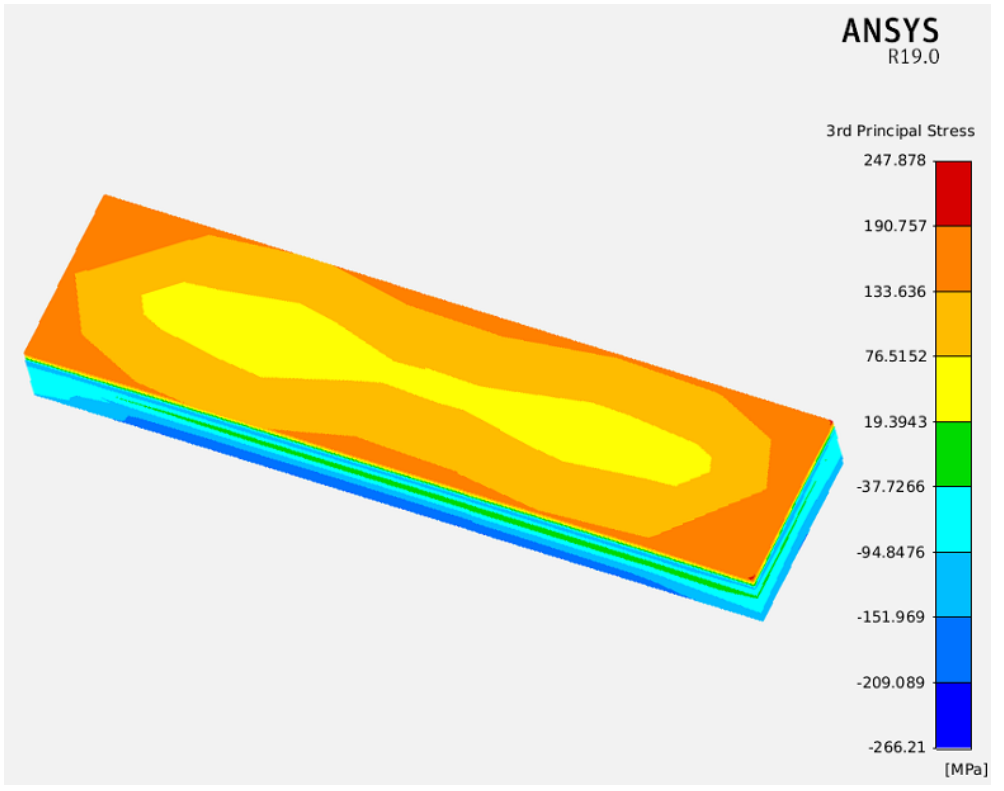

Figure 4. The residual stresses on the surfaces of a quarter of the 6061 aluminium alloy plate after the quenching process numerical values.

During the quenching process different cooling takes place on the bottom vs the top of the plate, also, a similar behavior is observed for the residual stresses with higher absolute values recorded at the bottom of the plate (Figure 5). 


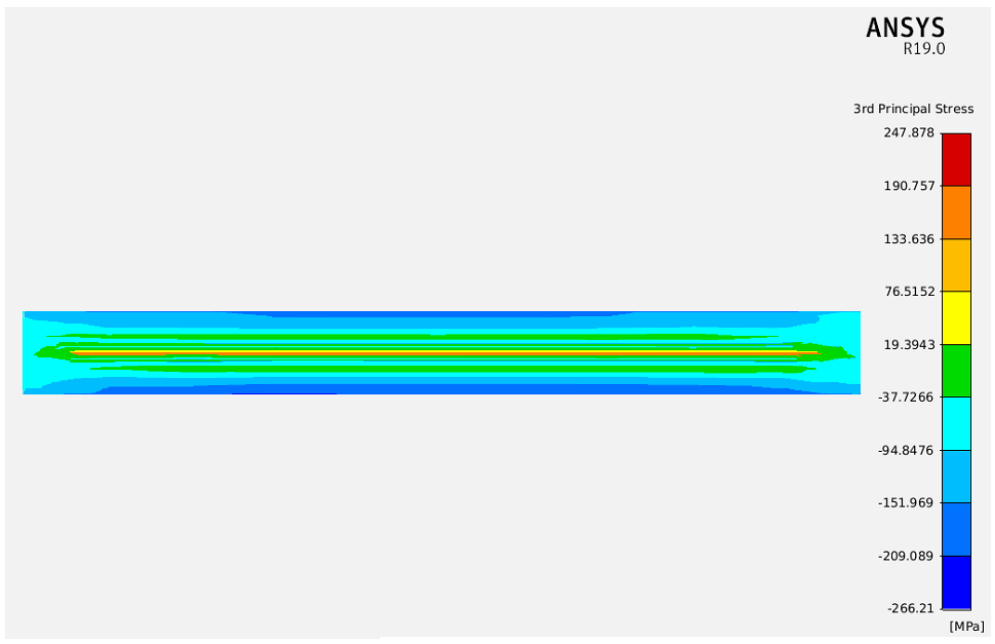

Figure 5. The residual stresses on the transversal section view of the 6061 aluminium alloy plate after the quenching process numerical values. Different values were obtained on the bottom of the plate vs the top of the plate.

From this figure can been seen that the residual stresses after quenching process follow an M-shaped profile on the depth of the plate. The determined profile of the axial residual stresses by using Integral Method is presented in Figure 6.

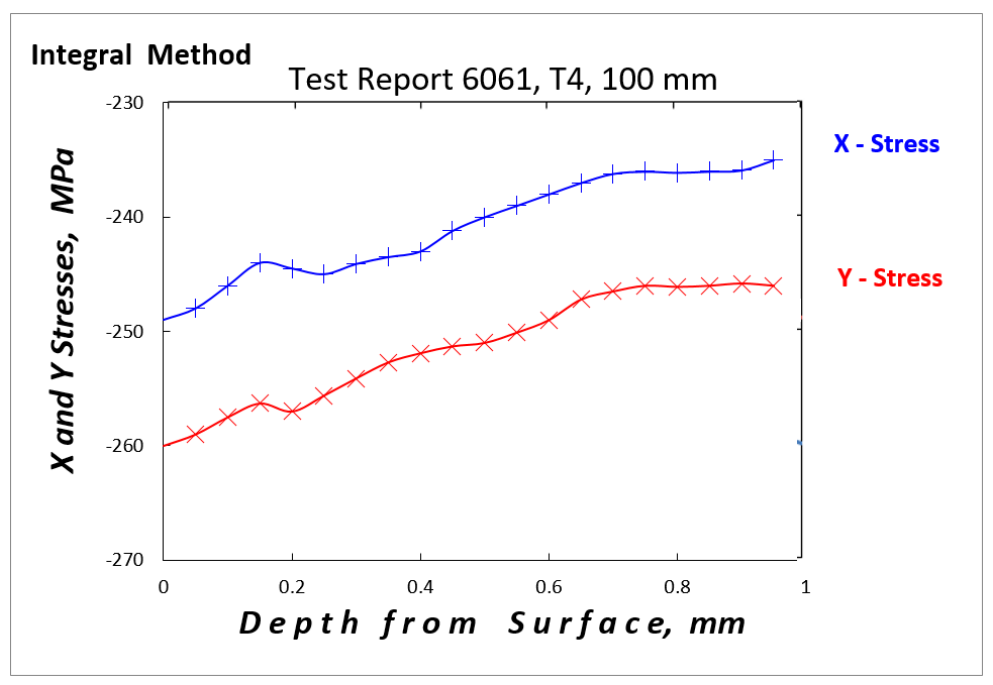

Figure 6. Non-uniform stress profile determined for 6061 aluminium plate after quenching process.

The values obtained for the residual stresses on the bottom surface of the plate were similar to those obtained by using ANSYS software. 


\section{Conclusions}

The numerical results were obtained by developing and implementing a mathematical model in ANSYS Discovery AIM 19.0 software.

The temperature of the transient heat transfer problem between the demineralized water and the 6061 aluminium alloy plate was used as input data in the structural problem.

The residual stresses due to solid thermal effects for an aluminium plate were determined by calculation of the Third principal stresses. The numerical results obtained for the residual stress profile in the cross section of the aluminum alloy plate are in accordance with the theory, following an M-shaped profile.

The numerical values for the residual stresses obtained on the bottom surface of the plate were in the range of those obtained by using the Integral Method.

The possibility of performing simulations with different input data in the mathematical model gives a better understanding and control of residual stresses in aluminum alloy plates.

\section{Acknowledgement}

Part of the cost of the industrial equipment used to obtain the results presented in this work was funded by European Union through Competitiveness Operational Programme, Priority Axis 1 Research, Technological Development and Innovation, within the project Investments in the RED Department of ALRO aiming at improving the research infrastructure for the aluminium alloy heat treated plates with high qualification industrial applications, based on the Funding Contract no. 42/05.09.2016.

\section{References}

[1] P. Rashed and M.M.A. Hossain, Control of Distortion in Aluminium Heat Treatment, Chapter 13, Fundamentals of Aluminium Metallurgy, Recent Advances (Woodhead Publishing Series in Metals and Surface Engineering, 2018) 495-524.

[2] M.B. Prime and M.R. Hill, Residual stress, stress relief, and inhomogeneity in aluminum plate, Scripta Materialia 46(1), 77-82 (2002).

[3] J. Mackerle, Finite element analysis and simulation of quenching and other heat treatment processes: A bibliography (1976-2001), Computational Materials Science 27(3), 313-332 (2003).

[4] J.S. Robinson, D.A. Tanner, and C.E. Truman, The origin and management of residual stress in heat-treatable aluminium alloys, Strain 50(3), 185-207 (2014).

[5] M. Petre et al., Mathematical Modelling of the Quenching Process of 6061 Aluminium Alloy Plates, International Conference on Applied Mathematics and Numerical Methods (ICAMNM) - third edition October 29-31, 2020, Craiova, Romania.

[6] P.T. Summers, Y. Chen, C.M. Rippe, B. Allen, A.P. Mouritz, S.W. Case, and B.Y. Lattimer, Overview of aluminum alloy mechanical properties during and after fires, Fire Science Reviews 4(3) (2015).

[7] ASM Handbook Volume 2: Properties and Selection: Nonferrous Alloys and SpecialPurpose Materials (ASM International, 1990) $1328 \mathrm{pp.}$

[8] J. Wolberg, Data Analysis Using the Method of Least Squares: Extracting the Most Information from Experiments (Springer-Verlag, New York, 2005) 263 pp.

[9] P.J. Withers and H. Bhadeshia, Residual stress-II: nature and origins, Mater. Sci. Tech. 17(3), 66-375 (2001). 
[10] G.S. Schajer, Measurement of Non-Uniform Residual Stresses Using the Hole-Drilling Method, Journal of Engineering Materials and Technology 110(4), Part I. Stress Calculation Procedures, 338-343, Part II. Practical Application of the Integral Method, 344349 (1988). 